\title{
Hallazgo radiológico infrecuente por una hiperpresión abdominal tras un traumatismo
}

\author{
María del Mar Díaz Alcázar, ${ }^{1}$ Alicia Martín-Lagos Maldonado, ${ }^{1}$ Antonio Sáenz Gutiérrez, ${ }^{2}$ \\ Cristina Lozano Cejudo 3 \\ ${ }^{1}$ Hospital Universitario Clínico San Cecilio. Granada, España. \\ ${ }^{2}$ Hospital Virgen de Altagracia. Ciudad Real, España. \\ ${ }^{3}$ Hospital General Universitario de Ciudad Real. Ciudad Real, España.
}

Acta Gastroenterol Latinoam 2021;51(1):41

Recibido: 07/10/2020 / Aceptado: 26/02/2021 / Publicado online el 22/03/2021 / https://doi.org/10.52787/hmyj6165

\section{Caso clínico}

Un hombre de 33 años, sin antecedentes de interés, presenta un traumatismo craneoencefálico y abdominal tras un accidente en moto. A su llegada a nuestro centro se encuentra

Figura 1. Corte transversal de la tomografia computarizada abdominal en el que se observa gas en la vena mesentérica superior.

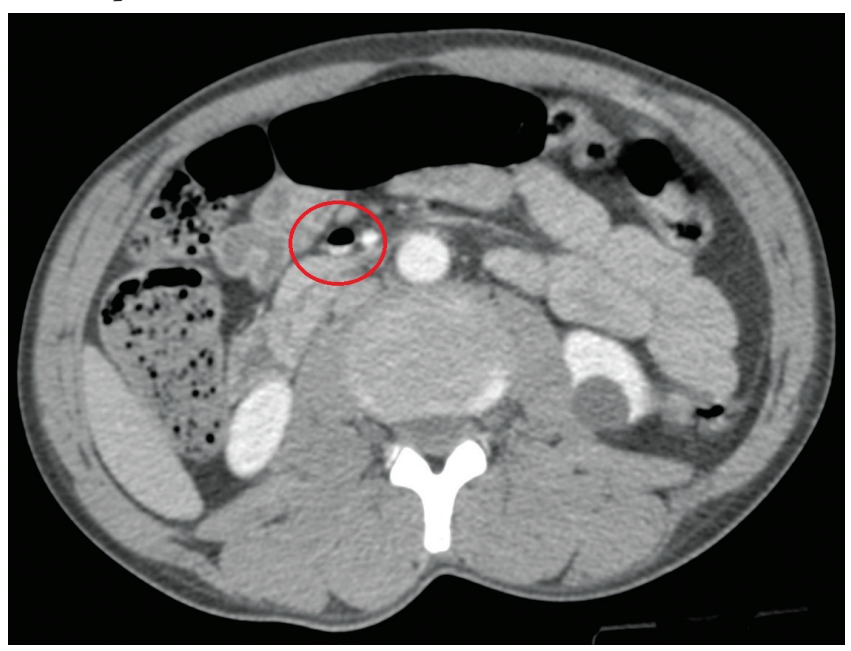

estable hemodinámicamente. La exploración abdominal es anodina. Analiticamente no hay hallazgos de interés. De todas formas, se le realiza una tomografía computarizada abdominal con contraste para descartar lesiones secundarias a un traumatismo abdominal observándose (Figuras 1 y 2).

Figura 2. Corte transversal de la tomografía computarizada abdominal en el que se reconoce gas en la vena porta y los radicales portales periféricos.

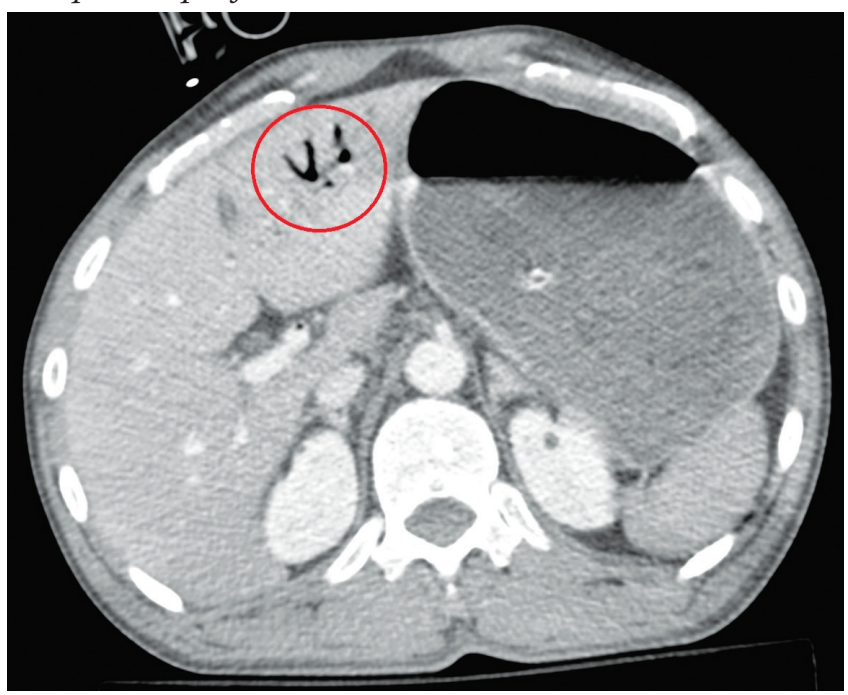

Correspondencia: María del Mar Díaz Alcázar Correo electrónico: mmardiazalcazar@gmail.com
¿Cuál es su diagnóstico? Resolución del caso en la página 118 


\title{
Solución del caso. Hallazgo radiológico infrecuente por una hiperpresión abdominal tras un traumatismo
}

\author{
Viene de la pagina 41
}

\section{Resolución}

En la tomografía computarizada abdominal, se identifica gas en la vena mesentérica y la circulación portal (Figuras 1 y 2). Dado que no hay otros signos de afectación orgánica se decide mantener una actitud expectante. El paciente permanece estable desde el punto de vista abdominal, pero acaba falleciendo a causa del traumatismo craneoencefálico.

La neumatosis intestinal es un hallazgo radiológico infrecuente consistente en la presencia de gas en la submucosa o subserosa, fundamentalmente del intestino delgado. ${ }^{1,2}$ Puede ser primaria, idiopática benigna; o secundaria $(85 \%)$, a enterocolitis necrotizante o isquemia mesentérica, entre otros. ${ }^{1-3}$ Puede asociarse a una neumatosis portal, lo que es aún más infrecuente. ${ }^{2}$

Las teorías etiopatogénicas son mecánica e infecciosa. ${ }^{1,2}$ Un traumatismo abdominal produce la compresión brusca y el aumento de la presión del gas intestinal intraluminal que puede lacerar la mucosa. ${ }^{1-3}$ La teoría infecciosa implica que los bacilos formadores del gas entren en la submucosa. ${ }^{1}$

La neumatosis no es una indicación obligatoria de laparotomía, ya que la ausencia de peritonitis puede permitir una actitud expectante. ${ }^{2} \mathrm{La}$ afectación de la mucosa no implica necesariamente necrosis. ${ }^{3}$
Aviso de derechos de autor

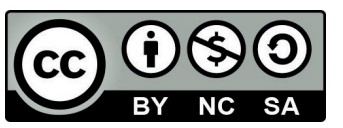

(C) 2021 Acta Gastroenterológica Latinoamericana. Este es un artículo de acceso abierto publicado bajo los términos de la Licencia Creative Commons Attribution (CC BY-NC-SA 4.0), la cual permite el uso, la distribución $y$ la reproducción de forma no comercial, siempre que se cite al autor y la fuente original.

Cite este artículo como: Díaz Alcázar MM, Martín-Lagos Maldonado A, Sáenz Gutiérrez A y col. Hallazgo radiológico infrecuente por una hiperpresión abdominal tras un traumatismo. Acta Gastroenterol Latinoam. 2021;51(1):41, 118. https://doi.org/10.52787/hmyj6165

\section{Referencias}

1. Di Grezia G, Gatta G, Rella R, Donatello D, Falco G, Grassi R, Grassi R. Abdominal Hernias, Giant Colon Diverticulum, GIST, Intestinal Pneumatosis, Colon Ischemia, Cold Intussusception, Gallstone Ileus, and Foreign Bodies: Our Experience and Literature Review of Incidental Gastrointestinal MDCT Findings. Biomed Res Int. 2017;2017:5716835.

2. Gurland B, Dolgin SE, Shlasko E, Kim U. Pneumatosis intestinalis and portal vein gas after blunt abdominal trauma. J Pediatr Surg. 1998;33(8):1309-11.

3. Deutsch SA, Christian CW. Pneumatosis Intestinalis Due to Child Abuse. Pediatr Emerg Care 2019;35(2):e32-3. 